\title{
Triggering on emerging jets
}

\author{
Dylan Linthorne $\odot^{*}$ and Daniel Stolarskiఠ ${ }^{\dagger}$ \\ Ottawa-Carleton Institute for Physics, Carleton University, 1125 Colonel By Drive, \\ Ottawa, Ontario K1S 5B6, Canada
}

(Received 6 April 2021; accepted 16 July 2021; published 20 August 2021)

\begin{abstract}
Confining dark sectors at the $\mathrm{GeV}$ scale can lead to novel collider signatures including those termed emerging jets with large numbers of displaced vertices. The triggers at the LHC experiments were not designed with this type of new physics in mind, and triggering can be challenging, especially if the mediator is relatively light and/or has quantum numbers such that additional jets are not automatically produced in each event. We show that the efficiency and the total event rate at current triggers can be significantly improved by considering initial state radiation of the events, with the largest increase in rate coming from simulation of two additional jets. We also explore possible new triggers that employ hit counts in different tracker layers as input into a machine learning algorithm. We show that these new triggers can have reasonably low background rates, and that they are sensitive to a wide range of new physics parameters even when trained on a single model.
\end{abstract}

DOI: 10.1103/PhysRevD.104.035019

\section{INTRODUCTION}

Confining hidden sectors with a confinement scale around the $\mathrm{GeV}$ scale have received significant attention for potential discovery at colliders [1-8] (for a recent review, see Chap. 7 of [9]), building on the seminal hidden valley work [10-12]. Besides providing interesting signatures at colliders, they can also be motivated by asymmetric dark matter [13-15] and by neutral naturalness [16-19]. This has led to several phenomenological studies at the LHC [20-23], flavor experiments [24], and future proposed experiments $[25,26]$. There is also an experimental search [27] that puts direct limits on certain regions of parameter space.

If the dark confining sector has a mediator to the standard model (SM) whose mass is much larger than the confining scale, then the lifetime of the lightest dark hadrons that are not stable will be parametrically larger than their inverse mass. One particularly interesting region of parameter space is where the lifetime of the decaying dark hadrons is in the millimeter to meter range, leading to spectacular signatures at the LHC's detectors [1]. For example, if the dark gauge group is QCD-like, then when dark quarks are produced, they will shower and hadronize, producing

\footnotetext{
dylan.linthorne@carleton.ca

stolar@physics.carleton.ca
}

Published by the American Physical Society under the terms of the Creative Commons Attribution 4.0 International license. Further distribution of this work must maintain attribution to the author(s) and the published article's title, journal citation, and DOI. Funded by SCOAP. dark jets. This is analogous to the production of ordinary quarks at a high energy lepton collider which then produce SM jets. Each of the dark hadrons will decay at a different position in the detector, and the energy of the dark jet will "emerge" into the detector. This signature was thus termed an emerging jet [1]. At distances long compared with the typical $c \tau$ of the dark hadrons, the dark jet will look like a QCD jet.

Motivated by the asymmetric dark matter scenario of [13], the work in [1] considered a scalar mediator $X$ that is charged under QCD and dark QCD. That means that the dominant collider signal will be pair production of the mediator, and each collider event will contain two emerging jets and two SM jets. This is also the scenario experimentally constrained in [27]. Another well-motivated possibility is the one considered in the original hidden valley literature [10-12]: a vector mediator $Z^{\prime}$ that couples to both quark and dark quark currents. A third possibility, the one expected in the neutral naturalness scenarios [16-19], is the SM Higgs or another scalar as the mediator $(H)$ to the dark sector. In both of the latter cases, we see two important differences between the original case of the $X$ :

(i) The production cross section of dark hadrons is a free parameter and not set by pair production via SM QCD.

(ii) The production, at leading order, does not include the associated production of SM jets in the hard event.

These cases are therefore more challenging experimentally.

A particularly important challenge with exotic signatures at the LHC is triggering. The event rate at the LHC is extremely high, and a trigger is employed to reduce the 
event rate by several orders of magnitude and attempt to record all the events of interest. The triggers were designed to be extremely efficient on many types of events and new physics models, but they are not designed for more exotic scenarios such as emerging jets. In the case of the $X$ mediator, if its mass is $\mathcal{O}(\mathrm{TeV})$, then the associated jets in combination with the very high total energy in the event make triggering relatively straightforward.

In this work we study the significantly more difficult case of triggering on the $Z^{\prime}$ mediator, focusing on the relatively lower mass regime, $M_{Z^{\prime}} \sim 100 \mathrm{GeV}-1 \mathrm{TeV}$. We will take a two-pronged approach. First, we will consider how well current triggers can capture these events and explore how the addition of initial state radiation can increase the efficiency. Using initial state radiation (ISR) to leverage an experimental search is now a commonplace strategy for various searches for new physics, with phenomenological analyses for supersymmetric models [28-31] going back nearly 40 years. This strategy has also been used to search for model-independent effective operators [32-34], and it is colloquially known as the mono- $X$ strategy. Experiments at the LHC have used the presence of a single high transverse momentum associated objects to search for various new physics models. Initially, these methods focused on effective models [35,36], and they are now used extensively to search for invisible states. A broad range of recent examples include monojet [37], monophoton [38], mono- $W$, and mono- $Z$ [39], placing constraints on many different types of models. In this work we show that both QCD and electroweak radiation can increase the trigger efficiency and increase the total number of events recorded, but ultimately QCD will lead to a higher rate than electroweak radiation.

The second strategy we employ is to consider new triggers using modern machine learning (ML) techniques. The landscape of machine learning applications within particle physics is becoming ever broader and more complex. Its utilities ranges from substructure classifications [40], such as jet discrimination [41,42], adjustments to particle flow algorithms using images of calorimeter responses [43], to multivariate analysis techniques explored at both CMS and ATLAS as the LHC moves toward the energy and intensity frontiers $[44,45]$. We look to investigate novel triggers based on simple machine learning methods that can be applied to the triggering stream. Complementary studies include [46] where a deep neural net is implemented at L1, resulting in high trigger efficiencies for a HL-LHC $15 \mathrm{KHz}$ target output, as well as the study of a more traditional trigger for displaced vertices [47]. CMS (ATLAS) has been conducting analyses of this type on low level information reconstructed from the triggering stream under the guise of data scouting (online trigger analysis) [48-50].

One possible downside in the use of ML techniques in particle physics is that we might not understand the unique physical features that the algorithm is converging on [51]. We can easily use these methods as a black box while taking for granted the physical insight we might be losing out on. Some work has been done to try to mine features in these black boxes [52]. This is a significantly less important problem when considering triggers where the most important task is to get interesting events recorded quickly. Emerging jets contain complex dark substructures between the invisible dark sector hadrons and the visible SM particles they decay into. The question of "what aspect of the dark substructure is the machine learning," for example, to distinguish signal from background, is irrelevant in situ. The dataset, once written off-line, can be pruned for features using more modern "human readable" methods [53]. With that in mind, all use of ML methods in this paper will take a pragmatic approach, where we focus on writing as many interesting events onto record with little regard for physical insight.

In this work, we use hits in the tracker as input into a potential new trigger discriminator. Tracking is traditionally not used in triggering because track reconstruction is too computationally time consuming $[54,55]$. We skirt this problem by not reconstructing tracks, but rather by simply counting hits in a region of the detector. This is similar to the strategy proposed in [56] for $b$ tagging. This technique can be effective in distinguishing emerging jets because the dark sector particles will not leave hits but their decay products will. Therefore, if the dark sector particles lifetime $c \tau \sim$ millimeters to meters, an emerging jet will have increasing numbers of hits in detector layers further from the interaction point. QCD jets, on the other hand, will typically have the same number of hits in most layers because unstable SM hadrons will typically decay before hitting the first or second layer of the detector, with the exception of a few strange mesons. We will show that this type of observable fed into a machine learning algorithm can be an effective trigger for a wide class of model parameters. We also explore the universality of such strategies and show that a trigger trained on one parameter point can be sensitive to a broad swath of parameter space.

The remainder of this paper is structured as follows: in Sec. II we describe the concrete model we use for our analysis, and in Sec. III we describe our simulation pipeline. In Sec. IV we describe how the mono- $X$ strategy can be used to improve event collection with current triggers, and in Sec. V we outline how new triggers can also be used to explore new parameter space. Conclusions are given in Sec. VI.

\section{MODELS FOR EMERGING JETS}

The study of generic hidden sectors at the LHC is an interesting and important question. For concreteness we will specify a class of models and leave the more general case to future work. We consider an $S U\left(N_{d}\right)$ gauge group with confinement scale $\Lambda_{d} \simeq \mathrm{GeV}$ and $n_{f}$ flavors of 
vectorlike quarks with masses below confinement scale. The dark quarks are singlets under all SM gauge groups. If there is an accidental baryon number symmetry analogous to QCD, then the baryons of this sector could be dark matter [13].

Unlike previous work that studied a scalar mediator [1], we consider a vector mediator $Z_{\mu}^{\prime}$ as in some of the original hidden valley literature [10], whose mass $M$ is larger than the dark confinement scale, $M \gg \Lambda_{d}$. This is the simplest $s$-channel mediator to the hidden sector and can be thought of as a simplified model to parametrize more general mediator models. The UV theory and mechanism to give mass to the $Z^{\prime}$ is left unspecified, but we assume the additional states needed to not affect the emerging jet phenomenology. This assumption is justified by the separation of scales: the UV physics of the $Z^{\prime}$ is expected to be around the mass of the $Z^{\prime}$ and can easily be made heavier, while the emerging jet phenomenology is characterized by the scale $\Lambda_{d}$, which, as noted above, is small compared to the $Z^{\prime}$ mass.

This mediator couples to the quark and dark quark currents,

$$
\mathcal{L} \supset \frac{1}{2} M^{2} Z^{\prime \mu} Z_{\mu}^{\prime}+Z^{\prime \mu}\left(g_{q} \bar{q} \gamma_{\mu} q+g_{d} \kappa_{i j} \overline{Q_{i}} \gamma_{\mu} Q_{j}\right)
$$

where $q$ are SM quarks, $Q$ are dark quarks, and $g_{q / d}$ are coupling constants. The matrix $\kappa_{i j}$ is $n_{f} \times n_{f}$ and introduced to break the $S U\left(n_{f}\right)$ hidden flavor symmetry that would otherwise stabilize some of the dark pions. We do assume flavor universality for the coupling to SM quarks to avoid bounds from flavor physics, and SM flavor indices are summed and not written. The $Z^{\prime}$ is a singlet under SM and unbroken dark gauge groups, so gauge indices among the quarks and dark quarks are contracted in the standard way.

This model contains a rich spectrum of dark hadrons, with a multiplet of dark pions, $\pi_{d}$ expected to be the lightest. All heavier mesons have a lifetime of order $\Lambda_{d}^{-1}$ and decay to dark pions if kinematically allowed (i.e., $\rho \rightarrow$ $\pi \pi$ in the SM). Dark baryons in these models are often very long-lived. In the parameter regions we consider, dark pions are significantly lighter than dark baryons (as in QCD) and thus are typically produced in much larger abundances than dark baryons. This can be confirmed with SM data [57] as well as a large $N_{c}$ expansion [58] of QCD. Therefore, we ignore the effects of dark baryons in this study, but of course these assumptions can be violated if the hadron spectrum is significantly different from that of the SM.

For simplicity, we take a common mass of the dark pions $m_{\pi_{d}}$, and we assume there are no large hierarchies between the entries in the $\kappa$ matrix. These assumptions allow us to consider a common lifetime for all the dark pions, but for a study of nontrivial dark flavor dynamics, see [24]. The $Z^{\prime}$ mediates a decay of the dark pions that can be computed using dark chiral perturbation theory with a width given by

$$
\Gamma\left(\pi_{d} \rightarrow \bar{q} q\right) \approx \sum_{q} \frac{g_{q}^{2} g_{d}^{2} N_{c} m_{q}^{2} f_{\pi_{d}}^{2}}{32 \pi M^{4}} m_{\pi_{d}},
$$

where $N_{c}=3$ is the number of SM colors, $f_{\pi_{d}}$ is the dark pion decay constant, which we take to be $\sim \Lambda_{d}$, and $m_{q}$ is the mass of the SM quark in the final state. Here and in the formulas in the remainder of this section, we have assumed that the entries in the $\kappa$ matrix are $\mathcal{O}(1)$ and can be ignored for a leading-order estimate. The sum is over all SM quarks that are kinematically accessible, and we have ignored phase space effects, but they can be trivially added. The factor of $m_{q}^{2}$ is a spin-parity affect analogous to the decay of the charged pion in the SM, implying that the decay will be dominated by the heaviest quark kinematically accessible. We can estimate the proper decay length for a benchmark pion mass of $2 \mathrm{GeV}$,

$$
\begin{aligned}
c \tau_{0} \approx & 80 \mathrm{~mm} \times \frac{1}{g_{d}^{2} g_{q}^{2}} \times\left(\frac{2 \mathrm{GeV}}{f_{\pi_{d}}}\right)^{2} \\
& \times\left(\frac{100 \mathrm{MeV}}{m_{q}}\right)^{2}\left(\frac{2 \mathrm{GeV}}{m_{\pi_{d}}}\right)\left(\frac{M_{Z^{\prime}}}{1 \mathrm{TeV}}\right)^{4},
\end{aligned}
$$

which we see can be macroscopic but smaller than the size of an LHC detector for a wide range of parameter space.

Dark quarks (and thus dark jets) are produced at colliders like the LHC via an $s$-channel $Z^{\prime}$. If kinematically accessible, resonant production where the $Z^{\prime}$ is on shell will dominate the production. The lowest order cross section for this production process at a proton-proton collider is

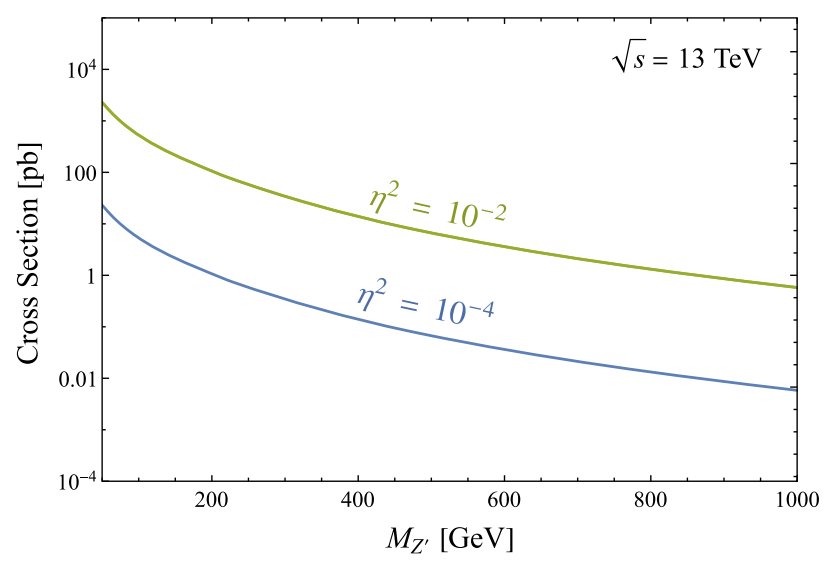

FIG. 1. Cross section of $p p \rightarrow Z^{\prime} \rightarrow Q \bar{Q}$ for a leptophobic $Z^{\prime}$ as a function of its mass $M_{Z^{\prime}}$. Generated by convolving the partonic cross sections of $u, d$ quarks with their respective parton distribution functions at a center-of-mass energy of $\sqrt{s}=13 \mathrm{TeV} . \eta$ is a function of the $Z^{\prime}$ Lagrangian parameters defined in Eq. (6). 


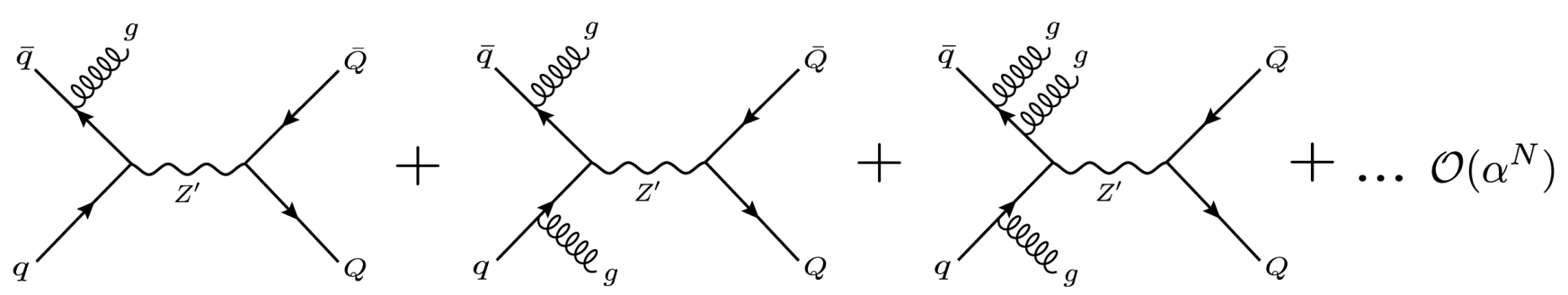

FIG. 2. Higher order QCD initial state radiation diagrams of $q \bar{q}$ induced $Z^{\prime}$ resonant production. Gluons are radiated from the quark lines in the form of detectable hard QCD jets. Electroweak (EW) splittings are also possible with diagrams scaling with the EW couplings.

$$
\begin{aligned}
\sigma\left(p p \rightarrow Z^{\prime} \rightarrow Q \bar{Q}\right)= & \sum_{f=u, d} \int d x_{1} f_{f}\left(x_{1}\right) \int d x_{2} f_{\bar{f}}\left(x_{2}\right) \\
& \times \frac{g_{d}^{2} g_{q}^{2}}{72 \pi}\left(\frac{x_{1} x_{2} s}{\left(x_{1} x_{2} s-M_{z^{\prime}}^{2}\right)^{2}+\Gamma^{2} M_{Z^{\prime}}^{2}}\right),
\end{aligned}
$$

where $f_{i}\left(x_{i}\right)$ is the parton distribution function for fermion $i$ and momentum fraction $x_{i}$. The Mandelstam variable $s$ is set to the center-of-mass energy $\sqrt{s}=13 \mathrm{TeV}$. The total decay width of the $Z^{\prime}$ is given by $\Gamma$, which has two contributions $Z^{\prime} \rightarrow q_{i} \bar{q}_{i}$ and $Z^{\prime} \rightarrow Q_{i} \bar{Q}_{i}$,

$$
\Gamma\left(Z^{\prime} \rightarrow X \bar{X}\right) \simeq \frac{N n g^{2} M_{Z^{\prime}}}{24 \pi}
$$

where for $X$ being a SM (dark) quark, $N$ is the number of (dark) colors which we take to be three, $n$ is the number of accessible flavors, and $g$ is the coupling to (dark) quarks. We have ignored kinematic factors that are only important if the $Z^{\prime}$ is approximately degenerate with a pair of (dark) quarks.

From these equations, assuming resonant production is dominant, we can show that to leading order the cross section for the process $q \bar{q} \rightarrow Z^{\prime} \rightarrow Q \bar{Q}$ depends only on the mass of the $Z^{\prime}$ and the variable

$$
\eta^{2} \equiv \frac{g_{d}^{2} \cdot g_{q}^{2}}{g_{d}^{2}+\left(\frac{n_{f}}{n_{d}}\right) g_{q}^{2}},
$$

scaling linearly with $\eta^{2}$. We have assumed the number of dark colors is also three. The production cross section at a center-of-mass energy $\sqrt{s}=13 \mathrm{TeV}$ is shown in Fig. 1 as a function of mass for a couple of benchmark values of $\eta^{2}$. We see that number of such events at the LHC with an integrated luminosity $\sim 100-3000 \mathrm{fb}^{-1}$ can be very large.

Alongside the leading-order (LO) contribution to the $Z^{\prime}$ production are higher order terms from ISR coming off of the incoming quark lines. In Fig. 2 we show this for QCD gluon radiation. These gluons will hadronize into additional hard QCD jets affecting the overall event topology. ISR is not exclusive to $\mathrm{QCD}$, the quarks may radiate a hard $W, Z$, or photon. These ISR contributions have a smaller rate than the leading-order process, but they can be easier to detect experimentally.

Independent of ISR, the choice of portal to the hidden sector can add stringent constraints on the mediator from collider and direct detection experiments [22], as was seen for the scalar mediator case explored in [1]. In our case, these $Z^{\prime}$ portals are of interest for detector searches because of the freedom in the production cross seōction of dark hadrons parametrized by the couplings to both the SM and the hidden sector $g_{q / d}$. This portal, however, does have resonant dijet production proportional to $g_{q}^{4}$, which can place constraints [59-61]. Most studies at modern collider experiments are only sensitive to the higher mass regime $M_{Z^{\prime}}>1 \mathrm{TeV}$, but some searches have employed in situ analysis to probe lower masses [62,63]. Typical upper bounds at the lower mass regime are $g_{q} \lesssim 0.1$. We do demand that the dark mesons have lifetimes between $1 \mathrm{~mm}$ and $1000 \mathrm{~m}$. Doing so puts additional constraints on the product of couplings $g_{d} \cdot g_{q}$ from Eq. (1). Assuming lower mediator masses, dark pion masses, and decay constant at $\mathcal{O}(\mathrm{GeV})$, emerging jet events with displaced vertices are consistent with $g_{d} \cdot g_{q} \lesssim 0.2$.

For the rest of this work, we take $c \tau$ to be a free parameter. The mass of the $Z^{\prime}$ and $c \tau$ are related by Eq. (3), but there are enough parameters in the models such that we can tune each variable independently. Therefore, we vary the mass of the $Z^{\prime}$ as it broadly controls the total energy in the event. For the dark QCD parameters, we use six

TABLE I. Dark sector parameters for our benchmark models. $\Lambda_{d}$ is the dark confinement scale, $m_{V}$ is the mass of the dark vector mesons, and $m_{\pi_{d}}$ is the pseudoscalar mass. $c \tau_{\pi_{d}}$ is the rest frame decay length of the pseudoscalars. We take $N_{c}=3$ and $n_{f}=7$ in both benchmarks. Models $\mathrm{A}$ and $\mathrm{B}$ are considered in Sec. IV, while models A, C, D, E, and F are considered in Sec. V.

\begin{tabular}{lcccccc}
\hline \hline Model & $\mathrm{A}$ & $\mathrm{B}$ & $\mathrm{C}$ & $\mathrm{D}$ & $\mathrm{E}$ & $\mathrm{F}$ \\
\hline$\Lambda_{d}$ & $10 \mathrm{GeV}$ & $4 \mathrm{GeV}$ & $10 \mathrm{GeV}$ & $10 \mathrm{GeV}$ & $10 \mathrm{GeV}$ & $20 \mathrm{GeV}$ \\
$m_{V}$ & $20 \mathrm{GeV}$ & $8 \mathrm{GeV}$ & $20 \mathrm{GeV}$ & $20 \mathrm{GeV}$ & $20 \mathrm{GeV}$ & $40 \mathrm{GeV}$ \\
$m_{\pi_{d}}$ & $5 \mathrm{GeV}$ & $2 \mathrm{GeV}$ & $5 \mathrm{GeV}$ & $5 \mathrm{GeV}$ & $5 \mathrm{GeV}$ & $10 \mathrm{GeV}$ \\
$c \tau_{\pi_{d}}$ & $150 \mathrm{~mm}$ & $5 \mathrm{~mm}$ & $50 \mathrm{~mm}$ & $500 \mathrm{~mm}$ & $5 \mathrm{~mm}$ & $500 \mathrm{~mm}$ \\
\hline \hline
\end{tabular}


benchmark parameter points shown in Table I. The first two, model A and model B, have different dark pion masses of 2 and $5 \mathrm{GeV}$ and two different lifetimes of 5 and $150 \mathrm{~mm}$, respectively. These models are used in studying current triggers in Sec. IV. ${ }^{1}$ Models C, D, and E, are identical to model A but with lifetimes ranging from 5 to $500 \mathrm{~mm}$. Model $\mathrm{F}$ has the same lifetime as model D but a heavier hadron spectrum. Models A, C, D, E, and F are considered in the machine learning trigger analysis of Sec. V.

\section{EVENT GENERATION}

Here we describe our simulation pipeline to generate Monte Carlo events for the models described in the previous section. The events were generated using a modified spin-1 mediator model ${ }^{2}$ [4], implemented using the FeynRules [64] package. The model is outputted as a Universal FeynRules Output [65] file, which allows generation of hard processes with Madgraph5_aMC@NLO [66], and we use LHC conditions with a center-of-mass energy of $13 \mathrm{TeV}$. This output is interfaced to the hidden valley $[67,68]$ module of PYTHIA8 [69], which simulates showering and hadronization in the dark sector as well as decays of dark hadrons to either other dark hadrons or to SM states. ISR in QCD or EW, i.e., jets, leptons, and EW gauge bosons, are included at leading order in the hard processes in Madgraph5_aMC@NLO, and we use PYTHIA8 to shower and hadronize SM quarks. The resulting hadrons are clustered into jets using the anti- $k_{t}$ algorithm [70] implemented in FASTJET [71], where the ATLAS tracker's pseudorapidity is $|\eta|<2.49$ and the jet angular parameter $R=0.4$.

Double counting of jets can occur when introducing ISR at the matrix element level and then subsequently hadronizing the partons in PYTHIA8. To avoid this, we use the Mangano (MLM) matching and merging procedure [72]. An XQCut of $M_{Z^{\prime}} / 10$ is used at the matrix element level, which forces the production of only partons with a minimum $K_{T}$ separation. Matching in PYTHIA8 is done by enforcing $\mathrm{QCu}>\mathrm{XQCu}$. For photon ISR, a minimum transverse momentum cut is placed on additional photon radiation of $P_{T}>10 \mathrm{GeV}$ (the default value in Madgraph5_aMC@NLO) to avoid soft and collinear divergences. We stress that our simulation of ISR is at leading order, and we do not attempt to resum logarithms of the threshold scale.

For the analysis of current triggers in Sec. IV, $10^{6}$ events are generated for each $Z^{\prime}$ mass within the range $[50,1500] \mathrm{GeV}$, with a step size of $50 \mathrm{GeV}$, for models A and B in Table I. A $Z^{\prime}$ width of $\Gamma_{Z^{\prime}}=M_{Z^{\prime}} / 100$ is used throughout. A crude detector volume cut is implemented at the PYTHIA8 stage. All particles that have not decayed outside of a cylinder of $(r=3000, z=3000) \mathrm{mm}$ are

\footnotetext{
${ }^{1}$ These were also the benchmarks used in [1].

${ }^{2}$ https://github.com/smsharma/SemivisibleJets.
}

considered stable. This cut was placed to mimic the detector volume out to the muon spectrometer. For the analysis of current triggers, background rates are already known and do not have to be estimated.

In Sec. V, the focus is on using a machine learning approach for novel triggers. We are less interested in hard ISR events and therefore use PYTHIA8's hidden valley production process $f \bar{f} \rightarrow Z_{v}$ processes instead of Madgraph5_aMC@NLO. Hits on the ATLAS inner tracker are used as discriminating variables. A proper detector simulation of the inner tracker is outside of the scope of this work, but we use a crude detector simulation with code used in [5], which encompasses the ATLAS tracker from the inner bilayer (IBL) to the transition radiation tracker (TRT). This detector simulation assumes simple models of energy loss through each thin layer of the detector. We use this custom simulation package because standard detector simulations used in phenomenological studies such as DELPHES do not attempt to simulate the response to displaced decays. An emerging jet registers various hits as function of the radial distance from the interaction point. Section V considers models A, C, D, E, and F, and we choose a $Z^{\prime}$ mass of $M_{Z^{\prime}}=500 \mathrm{GeV}$. This set of models spans a wide range of lifetimes while keeping all other model parameters equal.

When considering new triggers, we must also estimate the background. Various background processes are considered, but the backgrounds are dominated by $p p \rightarrow b \bar{b}$ events, which have high multiplicity and hadrons with longer lifetimes, producing many displaced vertices that can mimic an emerging jet signal. We simulate $10^{5}$ events of $g g \rightarrow b \bar{b}$ using PYTHIA8's heavy flavor hard $b \bar{b}$ processes. The inclusive background cross section $\sigma_{\mathrm{bkg}}$ is taken from the PYTHIA8 event generation stage, which is used to estimate the instantaneous background rate. Pileup is added to both signal and background events with PYTHIA8's minimum bias events. For each signal or background event, a number of minimum bias events are added randomly sampled from a Poisson distribution with mean of $\mu=50$, mimicking the run 2 conditions used in the trigger menus for the analysis of Sec. V. The LHC is expected to go through a series of upgrades leading for run 3 and beyond, allowing for higher luminosities. Estimates within the first phase of run 3 give mean pileup contributions as high as $\mu=80$ [73]. Advances in hardware and changes to trigger thresholds are expected to relax the effects of this increase of soft minimum bias events on future searches [74].

\section{CURRENT TRIGGERS}

A consequence of having high instantaneous luminosities, such as at the LHC, is the necessity of implementing triggering streams on specified event criteria. ATLAS/CMS produce event rates in the megahertz range, which is far too large to write every event onto record. Triggers were introduced to greatly reduce the event rate that is written 
TABLE II. The 2017 ATLAS triggers [75] used in Sec. IV analysis. Triggers are separated by L1 thresholds, HLT thresholds, and off-line selection criteria. Reconstructed jets used in the single jet, MET, and $H_{T}$ trigger have $R=0.4$. Thresholds with (*) must additionally satisfy the isolation cone criteria in Eq. (8). A lower level threshold is not given for the $H_{T}$ as we seed it from the lower level single jet trigger instead.

\begin{tabular}{lccc}
\hline \hline Triggers & Lower $(\mathrm{GeV})$ & Higher $(\mathrm{GeV})$ & Off-line $(\mathrm{GeV})$ \\
\hline Single jet & 100 & 420 & 435 \\
Single $\gamma$ & $20^{*}$ & 140 & 145 \\
Single e & $22^{*}$ & $26^{*}$ & 27 \\
Single $\mu$ & 20 & $26^{*}$ & 27 \\
MET & 50 & 110 & 200 \\
$H_{T}$ & $\ldots$ & 850 & $\ldots$ \\
\hline \hline
\end{tabular}

for off-line use, to a manageable $\sim 1 \mathrm{kHz}$. Although emerging jet experimental searches do exist [27], models with unique phenomenology such as emerging jets may not be visible to the current dedicated trigger sets used at ATLAS and CMS [54,55]. In this section, we quantify the efficiency of current triggers for emerging jet phenomenology.

In addition to leading-order production, we also study the effects of ISR on trigger efficiencies that come from Feynman diagrams of the type shown in Fig. 2. Multiple QCD jets (and/or electroweak gauge bosons) can modify the naive expectation of a two-pronged emerging jet scenario. Each additional hard object will change the event's topology from back-to-back scattering in the transverse plane. Emerging jets on their own provide unique detector signals that may have difficulty passing trigger selections in various parameter regions. We will show that the inclusion of hard SM objects, such as jets and leptons, increases the likelihood of passing the triggers. Numerical trigger thresholds used in ATLAS [75] and used for this analysis are shown in Table II.

\section{A. Description of triggers}

The trigger systems at both experiments are separated into two disjoint online subsystems: the low level hardware trigger system (L1), and the high level software trigger system (HLT). L1 primarily deals with low level information from energy depositions in the calorimeter towers and minimally reconstructed jet variables. Track reconstruction and jet algorithms are available at HLT triggers for more sophisticated triggering criteria. Here, we will describe the triggers that are relevant to our analysis.

\section{MET triggers}

Dark sector mesons with sufficiently long lifetimes $\tau_{d}$ will typically escape the detector before decaying and thus contribute to missing transverse energy (MET). Energy deposited within the calorimeter towers is reconstructed for MET calculations at both ATLAS and CMS. In the plane transverse to the beam, the transverse momentum $P_{T}$ is conserved with a zero net $P_{T}$, and thus this observable is sensitive to production of invisible particles. Energy deposition in each tower is summed and a transverse momentum vector is constructed using

$$
P_{T}=\sqrt{\left(\sum P_{x}\right)^{2}+\left(\sum P_{y}\right)^{2}} .
$$

Any nonzero contribution is taken to be MET $=\left|P_{T}\right|$.

Triggers cut on an event's MET according to the trigger menu thresholds in Table II, at both L1 and HLT. L1 thresholds are lower than the HLT, although the calculated MET might differ between reconstructed calorimeter tower energies at both levels. It is usually precise enough to assume that HLT is seeded from L1 with 100\% efficiency; therefore, we only consider the HLT efficiencies.

Typical $Z^{\prime}$ events, even if the dark hadrons have long lifetimes, tend to have relatively low MET because the two emerging jets are produced back to back, so there can be significant cancellation between them. Hard initial state radiation can qualitatively change this picture as shown in Fig. 3. With additional radiation, the two emerging jets are no longer collinear, and their MET will to some extent add. Furthermore, the additional radiation means that the energy of each jet will be larger (for fixed $Z^{\prime}$ mass), which also tends to increase the MET. Figure 3 shows QCD radiation, but the same logic applies to EW radiation.

\section{2. $\mathrm{H}_{T}$ triggers}

The $H_{T}$ trigger is a threshold on the scalar $P_{T}$ sum of the event's reconstructed objects. These triggers help reduce

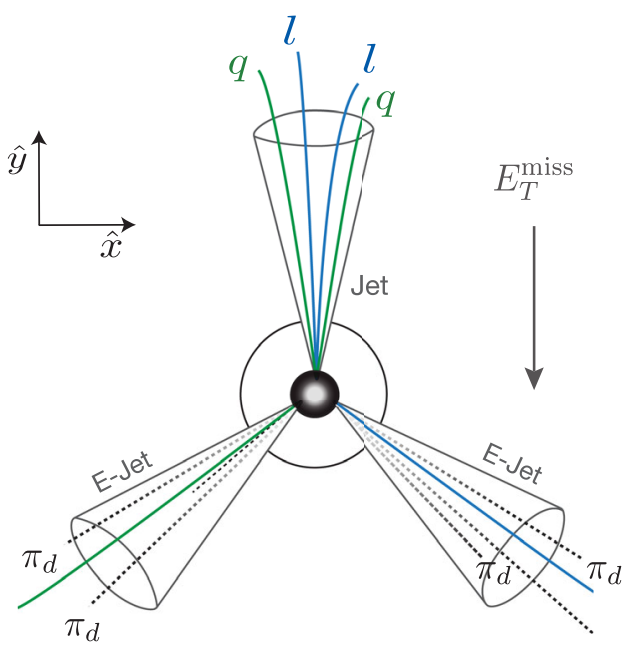

FIG. 3. The Mercedes topology of three jets recoiling off of each other within the $p_{T}$ plane. In this case, two emerging jets carrying missing transverse energy recoil off of a visible hard QCD jet. This configuration produces more missing transverse energy as the jet momentums are balanced in opposite directions. 
rates by focusing on events with large final state transverse energy $E_{T}$. The $H_{T}$ is built from objects with $|\eta|<2.5$ and jets are only included if they have $P_{T} \geq 50 \mathrm{GeV}$.

For the $Z^{\prime}$ model here, as the mediator mass increases, the trigger is more likely to be satisfied due to the larger final state momentum imparted onto the constituents. At lower mediator masses, the efficiency can be increased with additional hard QCD radiation. The QCD jets are more visible than the dark sector jets and lead to a higher $H_{T}$. This is also realized in the EW ISR case, as the hard photon $/ W / Z$ caries all of the ISR energy ${ }^{3}$ and, if properly reconstructed, contributes significantly to final state $E_{T}$. This effect can be seen in Fig. 3.

\section{Lepton and photon triggers}

ISR of a $W^{ \pm}, Z$ can add additional hard leptons and/or missing energy with neutrinos, and radiation of photons can also be used. Triggers that cut on identified leptons ( $e$ and $\mu$ ) and photons, are considered. The trigger menu hosts a range of triggers depending on the level of reconstruction necessary for the identification of the event's particles.

Leptons and photons in the trigger are required to pass isolation criteria. For leptons, ATLAS identifies uses various classifications. These criteria are known as tight (loose) isolation [76,77], which are defined by

$$
\sum_{i \in \text { cone }} \frac{P_{T}^{i}\left(\Delta R<R^{l}\right)}{P_{T}^{l}}<\mathcal{I},
$$

where in Eq. (8), $\mathcal{I}=0.6$ (1.5) for tight (loose) isolation, $P_{T}^{l}$ is the transverse momentum of the candidate lepton, $P_{T}^{i}$ are the transverse momentum of the visible noncandidate objects within the isolation cone, $\Delta R$ is the distance between the $i$ th particle and the candidate lepton $l$, and $R^{l}=0.2(0.3)$ is the cone radius for electrons (muons). This is accomplished with $P_{T}$ calculated from lepton track measurements.

In the case of photons, the isolation condition is given by

$$
\sum_{i \in \text { cone }} E_{T}^{i}\left(\Delta R<R^{\gamma}\right)<0.022 \cdot E_{T}^{\gamma}+2.45 \mathrm{GeV}
$$

The photon isolation uses calorimeter measurements of the transverse energy $E_{T}$ since photons do not leave tracks, $E_{T}^{\gamma}$ is the transverse energy of the candidate photon, and $E_{T}^{i}$ is the transverse energy of the $i$ th cone constituent. Since we are simulating events without full detector effects, we assume that the truth level transverse energy of the photon is equal to that of the reconstructed calorimeter energies.

\footnotetext{
${ }^{3}$ In leptonic decays of the $W$ or decays of the $Z$ to neutrinos, the energy of the neutrinos of course do not contribute to $H_{T}$.
}

Trigger menus may demand different levels of isolation strictness between L1 and HLT. In Table II, for the single electron trigger considered, both L1 and HLT must adhere to tight isolation criteria, whereas the muon trigger has isolation only at L1. It is important to consider these drastic differences of kinematic acceptance between L1 and HLT when calculating the total efficiency. Because of this, we do not assume that the lepton triggers are seeded from an L1 trigger with $\epsilon_{L 1}=1$ (100\% efficiency). Instead, we calculate the L1 efficiency and project the product $\epsilon=\epsilon_{L 1} \cdot \epsilon_{\mathrm{HLT}}$ in our results.

\section{B. Results with current triggers}

We first calculate the trigger efficiency for different triggers in model A and model B. In this section, we do not use a detector simulation as the output of PYTHIA should be a reasonable estimate of these simple variables. The efficiency is the number of events that pass the threshold, and therefore get written for off-line use, over the total number of events, and these are shown in Fig. 4, with the top (bottom) row being for model A (B). In the first column, we see that, for model A, any kind of radiation increases the MET trigger efficiency because in model A the dark hadrons have long lifetimes, and initial state radiation increases their momentum and makes them not back to back. Radiation of $W / Z$ does the best because of the presence of neutrinos. For model $B$, notice that we only get significant improvement with $W / Z$ radiation.

In the second column of Fig. 4, we see that QCD radiation can significantly increase the efficiency of the $H_{T}$ trigger for both lifetime benchmarks, and two hard jets do better than a single extra jet. Both $W / Z$ and photon radiation do better than QCD radiation because of the clean visible momentum carried by the EW radiation. At low masses $M_{Z^{\prime}} \lesssim 500 \mathrm{GeV}$, the efficiencies are similar for both models, while at higher masses $M_{Z^{\prime}} \gtrsim 500 \mathrm{GeV}$, model $\mathrm{B}$ becomes far more efficient as it carries more visible particles in the final state. The improvement due to extra radiation is very important at low mass, a section of the $Z^{\prime}$ parameter space not easily probed, but less so at high mass because the trigger can already be quite efficient at leading order in that case.

We also show the trigger efficiency as a function of dark pion lifetime for a fixed $Z^{\prime}$ mass of $800 \mathrm{GeV}$ in Fig. 5. As expected, as the lifetime increases, more of the energy escapes the detector and the MET trigger gets better, while the $H_{T}$ trigger gets worse. This explains the differences of the first and second columns of Fig. 4 between both models $\mathrm{A}$ and $\mathrm{B}$.

Finally, the third column of Fig. 4 considers the effect on lepton (photon) triggers on the process with additional $W / Z$ (photon) radiation. The two models are almost identical, showing that these triggers are picking up the extra electroweak radiation. This assures us that the dark pion lifetimes and parameters of the confined hidden sector 

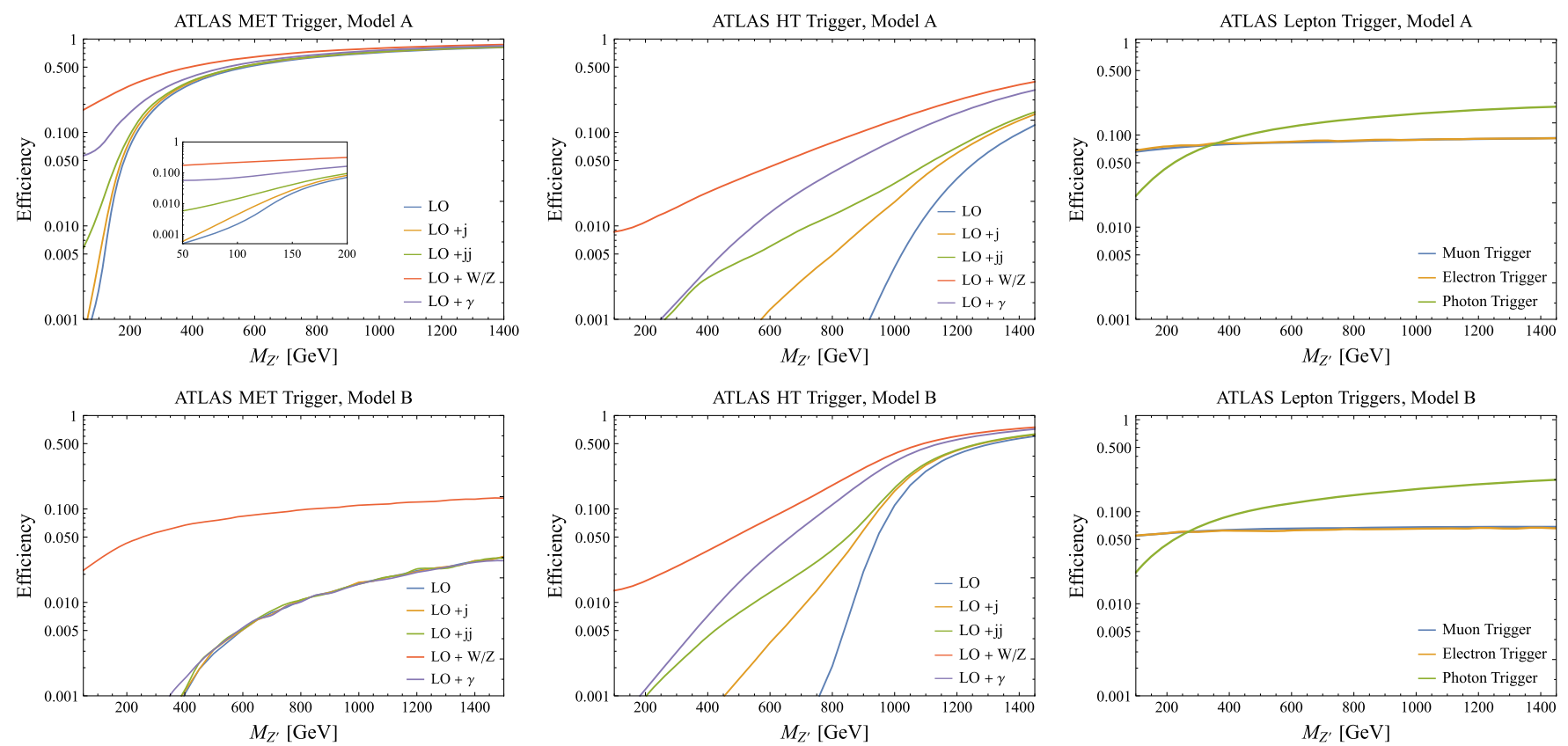

FIG. 4. ATLAS trigger efficiencies, at truth level, for various processes outlined in Sec. III. The first (second) row corresponds to model A (B) from Table I. In the first column, we have the MET triggers, the second column has the $H_{T}$ triggers, and, in the third column, we have the lepton and photon triggers. Both the electron and muon trigger efficiencies considered $W / Z$ ISR, while the photon trigger considered photon ISR only. All processes were generated under LHC conditions of a center-of-mass energy of 13 TeV. The trigger thresholds were taken from the ATLAS trigger menu summarized in Table II.

have little bearing on the EW focus of the lepton triggers. The slightly higher efficiency for lepton triggers $(e, \mu)$ in model $\mathrm{A}$ is because of the dark pion long lifetimes, leaving less contaminants within the lepton isolation cone, i.e., Eq. (8). In terms of the dependence on $M_{Z^{\prime}}$, the electroweak radiation is roughly constant, while the photons tend to be harder at larger $M_{Z^{\prime}}$, so the efficiency increases.

Although a useful metric, the efficiency does not consider the overall probability of the event occurring because

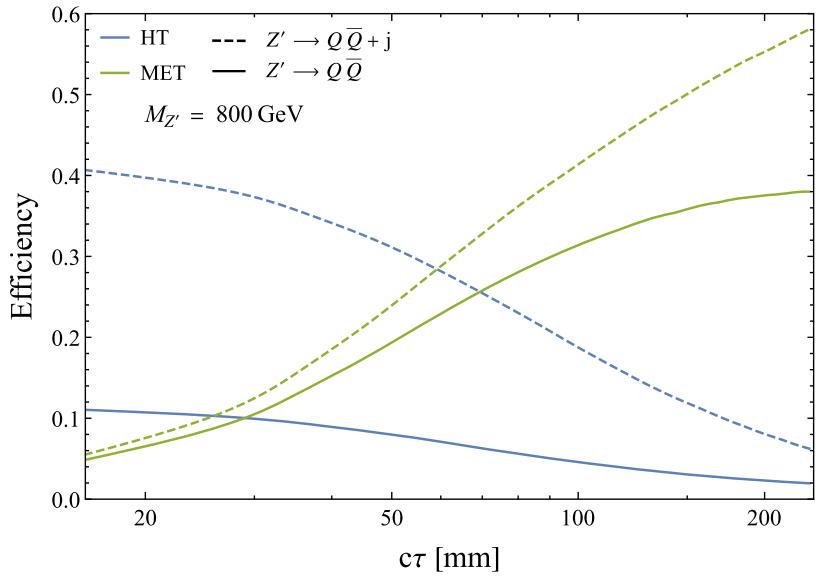

FIG. 5. ATLAS trigger efficiencies as a function of the dark pion lifetime $c \tau$. The LO process is shown in the solid lines, the $\mathrm{LO}+j$ process is shown in the dashed lines. An inverse relationship is exhibited between MET and $H_{T}$ efficiencies. it does not take into account that extra radiation reduces the cross section. Therefore, when looking at the total rate, we consider the cross section of the process times its respective efficiency. To get a proper sense of the rate, independent of some of the unknown particle physics such as the couplings of the $Z^{\prime}$, we take the ratio of the cross section times efficiency with respect to the LO result with no additional radiation and show the results in Fig. 6. In the left column we show the improvement achieved for the MET trigger in model A. We see that QCD radiation can lead to significant improvements at low mass, and even at high mass, simulating that extra radiation increases the overall rate by $\mathcal{O}(100) \%$. We also see that simulating two additional jets gives significant improvement relative to only a single jet at low mass. Electroweak radiation only gives a modest improvement in the event rate, roughly $10 \%$ at low mass and even more modest at high mass. This is because the rate suppression due to $\alpha$ is significantly stronger than that from QCD that goes like $\alpha_{s}$.

In the right column of Fig. 6, we show the improvement for the $H_{T}$ trigger in model $\mathrm{B}$. At low mass, none of the 400,000 events we simulate at leading order pass the trigger, so considering radiation opens a new parameter regime for discovery. Even at intermediate masses, $M_{Z^{\prime}} \sim 500 \mathrm{GeV}$, additional radiation gives orders of magnitude improvement in rate. As with model A, two additional jets give the greatest improvement, but all processes considered can be significant. 

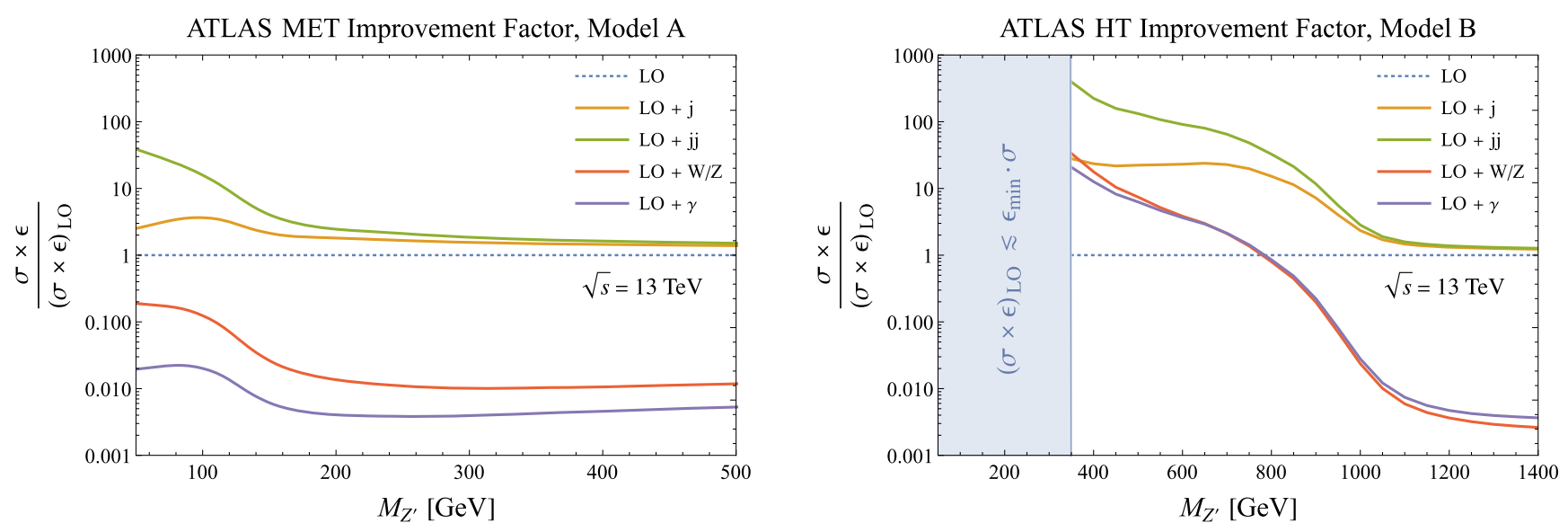

FIG. 6. Cross section times efficiency of various processes (leading-order, one-jet ISR, two-jet ISR, electroweak ISR) scaled by their respective leading-order process. The left plot uses the MET trigger and model A, while the right uses $H_{T}$ and model B. The dotted line is the leading-order process, which when normalized to itself is just unit as a reference for the rate boost obtained from additional processes. On the right plot, the blue region on the left has zero simulated events pass the trigger, $\epsilon \lesssim \epsilon_{\min }=1 / 400,000$.

\section{MACHINE LEARNING TRIGGERS}

The current triggers of Sec. IV, although well understood, are still limited in their capacity to find new physics. They have forced us to consider subsets of model parameter space that best produce specific signals that current triggers discriminate best on and create blind spots in other regions of parameter space. The experiments at the LHC have been investigating more novel ML methods to replace the cutbased methods used for most analysis. Examples include $W$ and top quark discrimination using boosted decision trees (BDTs) [78,79], QCD jet substructure using adversarial neural networks [80], and even alternatives to simulations of detector responses using generative adversarial networks [81]. These nonlinear methods are not limited to primary detector features, such as hard jets and charged particle tracks, as they can be trained (unsupervised) to converge on nonintuitive abstract features within an event.

We are interested in methods that can be employed at the triggering stage. Previous proposals advocated using BDTs as a fast and reliable high level trigger [82]. Similar to cutbased methods, ML algorithms can be trained on a array of new physics simulations, opening up the door for unique correlations between new physics models that could prove generic for a dedicated trigger. Additionally, the computational resources necessary in training and testing the ML method does not impact the resources in situ as the training/ testing stage is done prior to implementation on the trigger stream.

Emerging jets produce interesting signatures and are complimentary to other models consisting of hidden sector portals with dark showers. Examples include semivisible jets [2,4] and soft unclustered energy patterns [5], which exhibit extreme cases of a similar baseline theory to that of emerging jets. In this section, we employ the use of the ATLAS inner trackers in training ML methods for emerging jet signals for the purpose of triggering. Like the complimentary models mentioned above, emerging jets produce a wealth of uncharacteristic lifetimes as compared to the SM. Here we show that recruiting the inner tracker allows a ML algorithm to converge on discriminating features that exploit this gap in lifetimes between the new physics and the SM. Whether ML methods converge on generic features of new physics or those more specific to the training model, understanding what is the physics behind these ML features has been a long-standing question. Answering this question requires solving an inverse problem of the ML output and proves more and more difficult as ML methods such as neural networks (NNs) and BDTs become more complex, although there is some recent progress [53]. Fortunately, we can largely disregard this problem since the purpose of the trigger is to get as many interesting events onto record as possible. Anything written onto record can then be properly analyzed off-line. So we take the pragmatic approach to ML and focus our attention on producing the highest trigger efficiency independent of what it is "seeing."

As in Sec. IV, the trigger systems at both L1 and HLT are limited by their allocated computational resources. These triggering operations must be fast enough to reduce the input data stream to $\sim 1 \mathrm{kHz}$. This can be challenging when data from all areas of the detector package are simultaneously used within the triggering systems in some form or another. Fitting nonlinear algorithms, including those using ML methods, at the trigger level, can be taxing on the available resources. In this case, we look at low level variables such as hits on the tracking detectors and simple jet reconstructions from L1. This is similar to the strategy proposed in [56] in a different context. The lack of fully constructed particle tracks and momentum measurements allows for fully trained algorithms to operate quickly on the incoming data streams. 


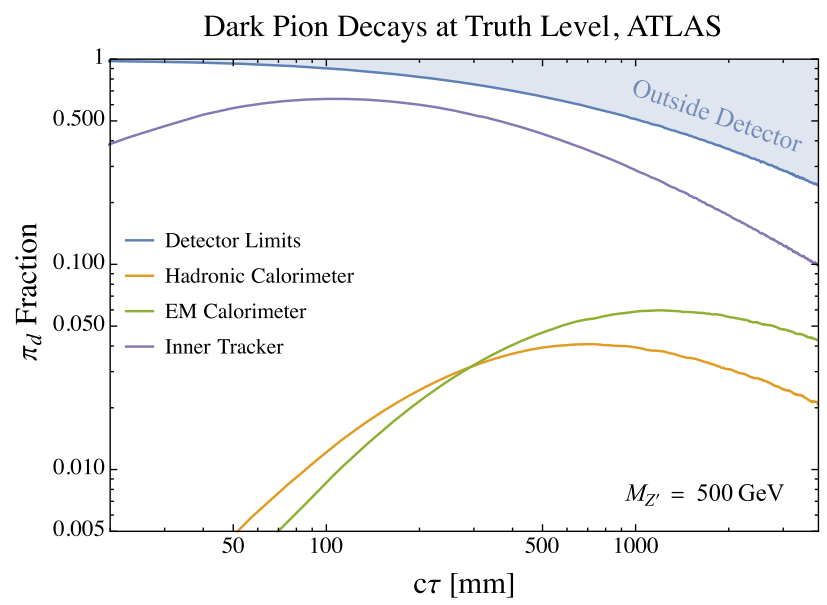

FIG. 7. The fraction of dark pions that decay into visible sector particles within the primary ATLAS detectors. The green line represents the decay fraction within the electromagnetic (EM) calorimeter, the orange for the hadronic calorimeter, and the blue for the inner trackers. The blue line is the fiducial boundary of the muon spectrometer, which we take to be the boundary of the ATLAS detector. We do not show that fraction that decays before the first tracker layer, and this fraction is significant at short lifetime. These fractions are computed from simulation with $M_{Z^{\prime}}=500 \mathrm{GeV}$.

For concreteness, we will analyze the ATLAS tracker geometry in this section, but we expect similar qualitative conclusions for CMS. In Fig. 7 we show the fraction of the dark pions that decay in a given detector subsystem as a function of lifetime, and we see that, for all lifetimes, the largest fraction is in the inner tracker. Therefore, we focus only on that system in this work, though we note that there could be interesting improvements by including the calorimeters and muon system.

The ATLAS inner tracker comprises, in increasing order of distance from the beam pipe, the inner bilayer, pixel detectors, silicon detectors, and transition radiation tracker. Table III shows the number of layers and radial distances from the interaction point, as well as whether the tracker has additional end caps. The primary purpose of the inner trackers is track reconstruction and particle identification. Tracks are reconstructed at the HLT level where calorimeter information has been seeded from L1. Although useful, track reconstruction is a very computationally expensive operation, as all possible track trajectories are backpropagated toward the interaction point. We propose using machine learning methods trained on the hit patterns of the inner tracker's layers while bypassing full track reconstruction.

As noted in Sec. III, in our simulations we take the dark hadronization to be dominated by dark pions. The proper lifetime of the dark pions depends strongly on the $Z^{\prime}$ mass as shown in Eq. (3), with lower mediator masses corresponding to shorter lifetimes. We assume a common lifetime for the dark pions, but for a study of hierarchical
TABLE III. ATLAS inner tracker specifications taken from $[83,84]$. The barrel layers of each tracker section are shown with their radial distance from the beam pipe/interaction point. Since the TRT is a more complex tracking package, we only consider the hits on the initial layer of the TRT. End caps also accompany most of the trackers, but are not considered in this analysis.

\begin{tabular}{lccc}
\hline \hline Tracker & Layer & Radius $(\mathrm{mm})$ & Geometry \\
\hline IBL & & 33.25 & Barrel \\
\hline \multirow{3}{*}{ Pixel } & First & 50.5 & \\
& Second & 88.5 & Barrel + End cap \\
& Third & 122.5 & \\
Semi conductor & First & 299 & \\
tracker (SCT) & Second & 371 & \\
& Third & 443 & Barrel + end cap \\
& Fourth & 514 & \\
TRT & Start & 554 & Barrel + end cap \\
\hline \hline
\end{tabular}

lifetimes see [24], and we take the lifetime to be a free parameter that we vary.

As the emerging jets traverse the inner tracker, the invisible dark pions decay into visible quarks that hadronize into SM jets with high particle multiplicity, creating a complex bundle of displaced tracks as they decay throughout the detector volume. Ideally, without pileup and other secondary detector effects, the number of hits registered on the tracker layers should increase with radial distance.

Signal and background events are simulated and explained in Sec. III. Hadronized events are passed to a highly simplified detector simulation used for [5] that attempts to model the ATLAS inner tracker. The simulation accounts for updated particle trajectories from the bending of the ATLAS toroidal magnetic field, $B \approx 2 \mathrm{~T}$, as well as energy loss from interactions with the material (assuming a thin layer approximation). The simulation does not account for the production of secondary particles from interactions with the layer materials. These secondaries are a possible source of error as they could fake a displaced vertex around each tracker. We are forced to use a custom simulation package because standard detector simulations used in phenomenological studies such as DELPHES do not track displaced vertices. For our analysis, we take combinations of the concentric tracker volumes utilizing the $\mathrm{IBL}_{i}$, $\operatorname{Pixel}_{i}$, $\mathrm{SCT}_{i}$, and $\mathrm{TRT}_{i}$ detectors where the subscript denotes the $i$ th layer. Results are represented as hit patterns in the form of heat maps in the $(\phi, \eta)$ plane for each layer of the trackers.

A simple strategy that ultimately does not work is to train a classifier using only the total number of hits recorded on each layer. This strategy reduces the 2D map in $(\phi, \eta)$ space to a single variable $N_{h, i}$ representing the number of hits on the $i$ th layer. The primary reason this strategy does not work is because of pileup. Each LHC event contains pileup from multiple proton collisions in each event. While there 

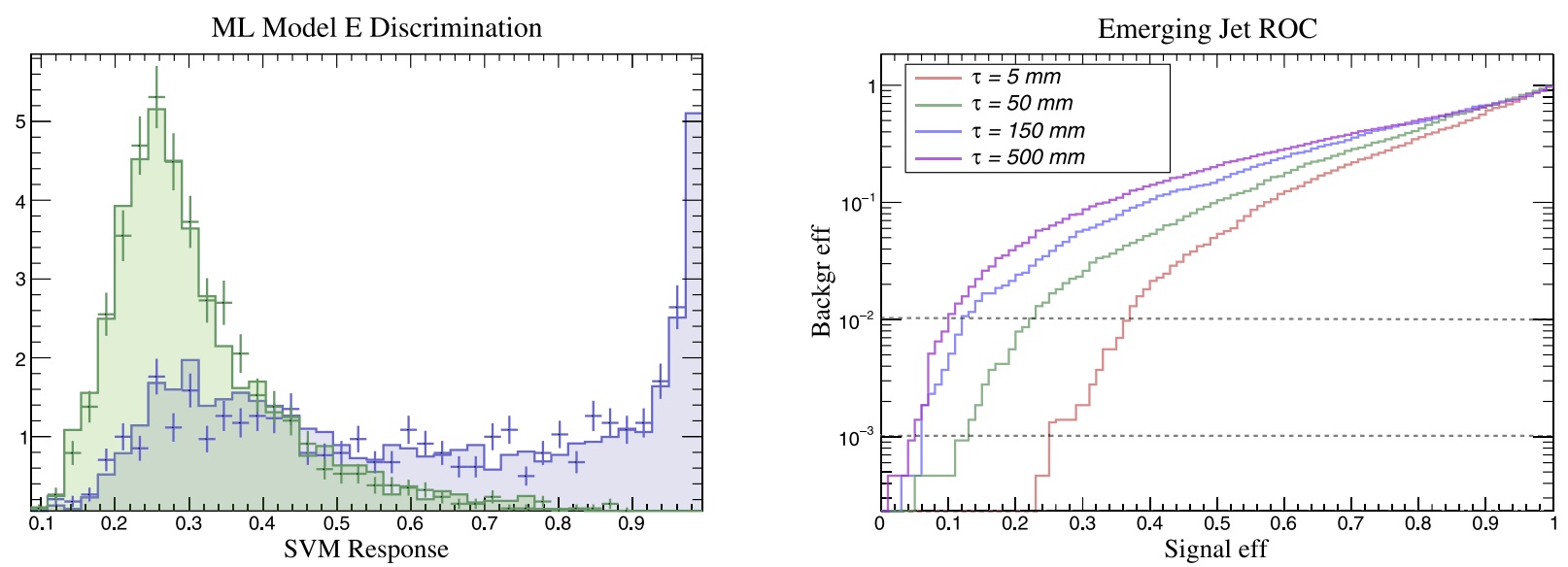

FIG. 8. On the left, model E (blue) discrimination from $b \bar{b}$ background (green) using a support vector machine. The SVM was trained using four layers IBL, $\mathrm{Pixel}_{2}, \mathrm{SCT}_{2}, \mathrm{TRT}_{1}$. An average pileup of $\mu=50$ was added to both signal and background. The flat bars (points) correspond to the training (test) set. On the right, Receiver Operation Characteristic ROC for models A, C, D, and E using the TMVA support vector machine. The SVM was trained and tested using four layers: IBL, $\mathrm{Pixel}_{2}, \mathrm{SCT}_{2}, \mathrm{TRT}_{1}$, with the input variable defined in Eq. (10). At a given background efficiency the expected signal efficiencies increase as the dark pion lifetimes lower. The required background rejection is estimated to lie between the horizontal dotted lines.

is only one hard collision responsible for the emerging jet production, the remaining collisions produce a large number of soft particles distributed approximately isotropically through the detector. This will typically wash out large differences in hits on consecutive layers $N_{h, i}-$ $N_{h, i+1} \approx 0$. As discussed in Sec. III, we simulate pileup by adding an average of 50 minimum bias collisions to both signal and $b \bar{b}$ background events. Since pileup is relatively soft, the toroid magnet will deflect charged particles with a radius of curvature inversely proportional to the particles momentum $R_{\mathrm{c}} \propto p^{-1}$. Pileup, in the form of minimum bias events, has characteristically low momentum spectrum, so layers at larger radial distances are less sensitive to pileup effects.

We refine our strategy by using the methods similar to those defined in [56], originally proposed for "trackless" $b$ tagging. The authors refer to their strategy to as "multiplicity jump," of using hit profiles on the tracking layers. They mitigate the issue of soft pileup contamination by selecting only the hits within a predefined jet cone axis. In their case, they applied a small jet cone radius of $\Delta R<$ 0.04 for $b$ tagging purposes. This makes calorimeter data inaccessible as the cone size is too small for the detectors' granularity.

We propose to generalize this to larger hadronic jets with $\Delta R<0.4$. By doing so, we can take advantage of low level calorimeter information, at trigger level, to calculate the jet cone directions of the event's hard objects. Subsequently, only hit multiplicities in the vicinity of these cones are considered hits. In detail, energy deposition in the calorimeter is used to reconstruct jets in terms of topoclusters at L1 and at HLT. With jet information at the trigger levels, we use the truth level jet vectors $\hat{v}$ as well as the jet cone acceptance $\mathcal{R}=0.5$ to define $N_{\text {cor }}^{i}$,

$$
N_{\mathrm{cor}}^{i} \equiv \sum_{j} N_{h, j}^{i}\left(\sqrt{(\Delta \eta)_{j}^{2}+(\Delta \phi)_{j}^{2}} \leq \mathcal{R}\right) .
$$

The sum runs through all grid points $j$ of the layer $i$. $(\Delta \eta)_{j}=\eta_{j}-\eta_{\hat{v}}$, where $\eta_{j}$ are pseudorapidities at grid point $j$ and $\eta_{\hat{v}}$ is the pseudorapidity of leading jet direction $\hat{v}$. Similarly, $(\Delta \phi)_{j}=\phi_{j}-\phi_{\hat{v}}$. The radius of each layer $R_{i}$ values can be found in Table III. This approach allows us to build a classifier that is sensitive to a conical subset of hit patterns in the direction of an L1 topocluster jet. This substantially reduces the $4 \pi$ reach of pileup.

We use the toolkit for multivariate data analysis toolkit [85] to train and test a ML discriminator. Multiple ML methods were tested, BDTs, NNs, and support vector machines (SVMs), and all methods investigated gave similar outputs for the level of this analysis. For that reason, we choose to use a SVM as our benchmark method, as it is the simplest method. The SVM was trained on events containing signal and $b \bar{b}$ background events.

For this analysis, we fix $M_{Z^{\prime}}=500 \mathrm{GeV}$. Models A, C, D, and $\mathrm{E}$ (described in Table I) are used as signal benchmarks. They vary in only the lifetimes, while keeping all other dark sector parameters equal. We also included model $\mathrm{F}$, which has the lifetime of model D but varies in the dark sector parameters. The TMVA support vector machine was trained and tested using Eq. (10) as the input variables. Testing and training sets were created and randomly separated from the simulation results. The left panel of Fig. 8 shows the resulting SVM signal and background discrimination. We see that there is excellent separation between signal background and that the trained and tested distributions look very similar. Only four layers of the trackers were used, IBL, Pixel $_{2}$, $\mathrm{SCT}_{2}, \mathrm{TRT}_{1}$, sampled from each of the four inner tracker packages. This arrangement of layers allows the ML to train 
on snapshots of the emerging jets evolution at substantially separated intervals, but using only four layers reduces the required size of the training sample. A proper study could simulate a much larger and realistic sample size, while incorporating additional layers.

Assuming that the event rate is background dominated, meaning very few new physics events occur, implemented triggers must reach background rates that do not exceed the allocated bandwidth. Unlike in Sec. IV where we investigated preexisting triggers, the background rates of our proposed novel triggers are unknown and must be estimated. The high level trigger rate usually allocated for new triggers is of $R \sim 1 \mathrm{~Hz}$ [86]. The required background rejection (Bkg rej) is given by

$$
\epsilon_{\mathrm{bkg}}=\frac{R}{\sigma_{\mathrm{bkg}} \mathcal{L}} \sim 10^{-3}-10^{-2}
$$

for a peak instantaneous luminosity of $\mathcal{L}=21 \times$ $10^{33} \mathrm{~cm}^{-2} \mathrm{~s}^{-1}$. The background rates were estimated assuming high $p_{T} b \bar{b}$ production, which primarily mimic the signal events. Additional backgrounds were considered, such as inclusive hard QCD backgrounds, generated through PYTHIA8. Since these additional background sources had substantially smaller efficiencies than pure heavy flavor $b \bar{b}$, we take them to be negligible to the total background rates. Inclusive background cross sections were taken from the PYTHIA8 hard event generation as explained in Sec. III, but these background cross sections are leading order and thus only order of magnitude estimates.

The new trigger background rejection rate will set an upper bound on the signal efficiency for each value of the ML response. The receiver operator characteristic curve (ROC) gives a visualization of this efficiency space. The ROC curve is shown in Fig. 8 as the background vs signal efficiency for each SVM response value. The value of the signal efficiency $\epsilon$ can be read off for each model at the highest allowable ATLAS trigger background efficiency. In Table IV, we see the range of $\epsilon$ for various lifetimes ranging from 5 to $500 \mathrm{~mm}$. Shorter lifetimes seem to outperform longer lifetimes. In model $\mathrm{E}\left(c \tau_{d}=5 \mathrm{~mm}\right)$ there are distinct hit patterns all within the IBL and the beginning of the TRT. These dark pions are boosted such that their respective emerging jets must go through the majority of its evolution between these inner tracker slices; whereas for the higher lifetime models, their evolution from invisible to visible is either skewed toward the later layers or even clipped beyond the tracker limits. Efficiencies of $\mathcal{O}(0.1-0.3)$ can be found for a comparable resonance mass of $M_{Z^{\prime}}=500 \mathrm{GeV}$ for the simple $s$-channel process with no additional hard ISR. Comparing these to the signal efficiencies found in Sec. IV (see Fig. 4) we see that, for similar topologies, training on detector hits can be advantageous, as current triggers have less reach in the low mass regime.

So far we have discussed training and testing on the same model parameters. Implementing a ML trigger would
TABLE IV. Signal efficiencies for expected allowable background rates for new ATLAS triggers for $M_{Z^{\prime}}=500 \mathrm{GeV}$. The lifetimes represent models A, C, D, and E. Each value in the last two columns are extracted from Fig. 8 for a background efficiency calculated using Eq. (11), assuming the events are fully saturated by background.

\begin{tabular}{lcc}
\hline \hline$c \tau_{d}(\mathrm{~mm})$ & $\epsilon\left(\right.$ Bkg rej $\left.10^{-2}\right)$ & $\epsilon\left(\right.$ Bkg rej $\left.10^{-3}\right)$ \\
\hline 5 & 0.370 & 0.250 \\
50 & 0.230 & 0.125 \\
150 & 0.122 & 0.060 \\
500 & 0.100 & 0.050 \\
\hline \hline
\end{tabular}

require training on some expected signal model prior to integration on the trigger stream. As mentioned earlier, emerging jets and other models of dark showers have a large available lifetime parameter space. Training a ML trigger on a single model parameter point could bias the trigger toward classifying only a small portion of this parameter space. The overall classification power is related to the AUC of the ROC curve. As a test of the universality of this method, we take an unknown sample set from each of the five models and apply each of the trained SVMs on them as was done in [87]. The results are seen in where the diagonal corresponds to the AUC of Fig. 9. Each row is of a SVM trained on a single lifetime and then applied to an unknown signal set of differing or same lifetime (columns). The deviations from each unknown lifetime set is of order of a few percent. This reinforces the insensitivity of the

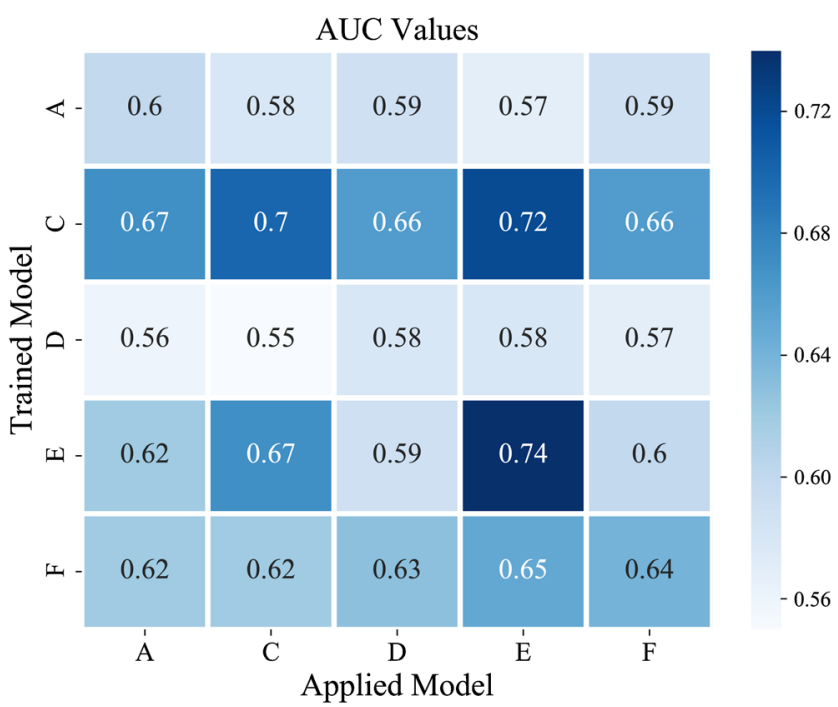

FIG. 9. Area under the curve (AUC) of the ROC curves. The SVM was trained on specific models (vertical axis) and tested/ applied on a unknown dataset (horizontal axis) of the same/ alternative model. The diagonals are the AUC values corresponding for training and testing on the same models, i.e., the ROC curves of Fig. 8. Trained models show little separation in classification power when applied to a range of lifetimes and hidden sector parameters. 
trigger to the parameters it was trained on. Each rows' average AUC value does not have a substantial change. Model F was included to see how sensitive this analysis is on the parameters of the hidden sector, such as the hadronization scale $\Lambda_{d}$ and the dark meson masses $\left(\pi_{d}, \rho_{d}\right)$. The first thing to notice is that when trained on model $\mathrm{F}$ there is almost no change in the AUC when applied to the range of lifetime models, much like model D, which shares the same lifetime. Second, when the various trained models are applied to both model $\mathrm{F}$ and model D, the AUC values are almost identical. This similarity gives us more confidence in the universality of the trigger, such that it is mostly sensitive to pion lifetimes, while being largely insensitive to other deviations in the hidden sector.

\section{SUMMARY AND CONCLUSIONS}

In this work we explore ways to trigger on new physics models with a high multiplicity of displaced vertex of the type explored in [1]. If the mediator is uncharged under the SM gauge groups, then it can be relatively light and associated SM radiation is not guaranteed, so triggering on these events at the LHC is not straightforward. If events are not triggered on, they are forever lost, so maximizing trigger efficiency is a necessary step for maximizing discovery potential.

We have first explored how both efficiency and event rate can be increased using current triggers with the addition of initial state radiation. Our main results are given in Figs. 4 and 6. Both QCD and electroweak radiation increase the total energy of the event and thus increases the efficiency of $H_{T}$ triggers. The effects are the largest for relatively light mediators, but they can also be relevant for heavy mediators. Other triggers, such as those searching for missing energy, leptons, and photons can also have higher efficiency with certain radiation. Processes with radiation have lower cross sections than the leading-order process, but we have shown that, even taking this into account, there are significant increases in event rate, especially at low mass. This ISR process is guaranteed by gauge invariance to exist, and we encourage experimentalists to simulate these processes in future studies.
We have also explored possible new triggers using modern machine learning methods that use simple counts of hits in the tracker as input variables. The new physics models considered here leave an increasing number of hits on each tracker layer as the dark mesons decay in flight to visible SM states. Counting hits in the tracker is significantly faster than performing track reconstruction, making it an ideal technique for a trigger. The use of ML techniques allows for a more sophisticated separation of signal and background based on these hit counts, and the effectiveness of our proposed method is shown in Fig. 8. Using ML for triggering can significantly reduce one of the primary problems of ML techniques in particle physics, that it is difficult to determine which features the algorithms are training on and thus difficult to estimate systematic errors. For a trigger, one wants to maximize the events recorded, and then a full study of systematics can be done at the analysis stage.

Finally, we have explored the sensitivity of our ML techniques across different model parameters as summarized in Fig. 9. Of course, the underlying model parameters of the new physics are unknown, and the ideal trigger would be sensitive to as much of the parameter space as possible and also to new physics models not captured by the simulation framework used in this work. We see that varying the particle physics parameters of the dark sector does significantly affect the efficiency, so a realistic trigger can be trained on one model parameter and still be sensitive to a broad class of new physics models.

\section{ACKNOWLEDGMENTS}

We thank Paul Archer-Smith, Kevin Earl, Dag Gillberg, Simon Knapen, Siddharth Mishra-Sharma, Michael Ramsey-Musolf, Pedro Schwaller, Jesse Thaler, and Andreas Weiler for helpful discussions. This work is supported in part by the Natural Sciences and Engineering Research Council of Canada (NSERC). D. L. acknowledges support from the NSERC Postgraduate Scholarships Doctoral Program (PGS D). D. S. is grateful for the hospitality from the Kavli Institute for Theoretical Physics and the Galileo Galilei Institute for Theoretical Physics where part of this work was done.
[1] P. Schwaller, D. Stolarski, and A. Weiler, J. High Energy Phys. 05 (2015) 059.

[2] T. Cohen, M. Lisanti, and H. K. Lou, Phys. Rev. Lett. 115, 171804 (2015).

[3] C. Csaki, E. Kuflik, S. Lombardo, and O. Slone, Phys. Rev. D 92, 073008 (2015).
[4] T. Cohen, M. Lisanti, H. K. Lou, and S. Mishra-Sharma, J. High Energy Phys. 11 (2017) 196.

[5] S. Knapen, S. Pagan Griso, M. Papucci, and D. J. Robinson, J. High Energy Phys. 08 (2017) 076.

[6] A. Pierce, B. Shakya, Y. Tsai, and Y. Zhao, Phys. Rev. D 97, 095033 (2018). 
[7] G. Burdman and G. Lichtenstein, J. High Energy Phys. 08 (2018) 146.

[8] H.-C. Cheng, L. Li, E. Salvioni, and C. B. Verhaaren, J. High Energy Phys. 11 (2019) 031.

[9] J. Alimena et al., J. Phys. G 47, 090501 (2020).

[10] M. J. Strassler and K. M. Zurek, Phys. Lett. B 651, 374 (2007).

[11] M. J. Strassler and K. M. Zurek, Phys. Lett. B 661, 263 (2008).

[12] T. Han, Z. Si, K. M. Zurek, and M. J. Strassler, J. High Energy Phys. 07 (2008) 008.

[13] Y. Bai and P. Schwaller, Phys. Rev. D 89, 063522 (2014).

[14] S. J. Lonsdale, M. Schroor, and R. R. Volkas, Phys. Rev. D 96, 055027 (2017).

[15] S. J. Lonsdale and R. R. Volkas, Phys. Rev. D 97, 103510 (2018).

[16] N. Craig, A. Katz, M. Strassler, and R. Sundrum, J. High Energy Phys. 07 (2015) 105.

[17] D. Curtin and C. B. Verhaaren, J. High Energy Phys. 12 (2015) 072.

[18] H.-C. Cheng, E. Salvioni, and Y. Tsai, Phys. Rev. D 95, 115035 (2017).

[19] C. Kilic, S. Najjari, and C. B. Verhaaren, Phys. Rev. D 99, 075029 (2019).

[20] M. Park and M. Zhang, Phys. Rev. D 100, 115009 (2019).

[21] T. Cohen, J. Doss, and M. Freytsis, J. High Energy Phys. 09 (2020) 118.

[22] H. Mies, C. Scherb, and P. Schwaller, J. High Energy Phys. 04 (2021) 049.

[23] S. Knapen, J. Shelton, and D. Xu, Phys. Rev. D 103, 115013 (2021).

[24] S. Renner and P. Schwaller, J. High Energy Phys. 08 (2018) 052.

[25] S. Alekhin et al., Rep. Prog. Phys. 79, 124201 (2016).

[26] D. Curtin et al., Rep. Prog. Phys. 82, 116201 (2019).

[27] A. M. Sirunyan et al. (CMS Collaboration), J. High Energy Phys. 02 (2019) 179.

[28] L. J. Hall and J. Polchinski, Phys. Lett. B 152, 335 (1985).

[29] O. Nachtmann, A. Reiter, and M. Wirbel, Z. Phys. C 27, 577 (1985).

[30] D. A. Dicus, S. Nandi, and J. Woodside, Phys. Rev. D 41, 2347 (1990).

[31] A. Brignole, F. Feruglio, M. L. Mangano, and F. Zwirner, Nucl. Phys. B526, 136 (1998); B582, 759(E) (2000).

[32] Y. Gershtein, F. Petriello, S. Quackenbush, and K. M. Zurek, Phys. Rev. D 78, 095002 (2008).

[33] Y. Bai, P. J. Fox, and R. Harnik, J. High Energy Phys. 12 (2010) 048.

[34] J. Goodman, M. Ibe, A. Rajaraman, W. Shepherd, T. M. P. Tait, and H.-B. Yu, Phys. Rev. D 82, 116010 (2010).

[35] V. Khachatryan et al. (CMS Collaboration), Eur. Phys. J. C 75, 235 (2015).

[36] G. Aad et al. (ATLAS Collaboration), Phys. Rev. Lett. 112, 041802 (2014).

[37] G. Aad et al. (ATLAS Collaboration), Phys. Rev. D 103, 112006 (2021).

[38] A. M. Sirunyan et al. (CMS Collaboration), J. High Energy Phys. 10 (2017) 073.

[39] A. M. Sirunyan et al. (CMS Collaboration), J. High Energy Phys. 07 (2017) 014.
[40] A. J. Larkoski, I. Moult, and B. Nachman, Phys. Rep. 841, 1 (2020).

[41] E. M. Metodiev, B. Nachman, and J. Thaler, J. High Energy Phys. 10 (2017) 174.

[42] E. M. Metodiev and J. Thaler, Phys. Rev. Lett. 120, 241602 (2018).

[43] F. A. Di Bello, S. Ganguly, E. Gross, M. Kado, M. Pitt, L. Santi, and J. Shlomi, Eur. Phys. J. C 81, 107 (2021).

[44] D. Guest, K. Cranmer, and D. Whiteson, Annu. Rev. Nucl. Part. Sci. 68, 161 (2018).

[45] A. Radovic, M. Williams, D. Rousseau, M. Kagan, D. Bonacorsi, A. Himmel, A. Aurisano, K. Terao, and T. Wongjirad, Nature (London) 560, 41 (2018).

[46] J. Alimena, Y. Iiyama, and J. Kieseler, J. Instrum. 15, P12006 (2020).

[47] Y. Gershtein, S. Knapen, and D. Redigolo, arXiv:2012 .07864 .

[48] S. Mukherjee (CMS Collaboration), Proc. Sci., EPSHEP2019 (2020) 139.

[49] D. Anderson (CMS Collaboration), Proc. Sci., ICHEP2016 (2016) 190.

[50] ATLAS Collaboration, Technical Report No. ATL-DAQPUB-2017-003, CERN, Geneva, 2017, http://cds.cern.ch/ record/2295739.

[51] S. Chang, T. Cohen, and B. Ostdiek, Phys. Rev. D 97, 056009 (2018).

[52] R. Shwartz-Ziv and N. Tishby, arXiv:1703.00810.

[53] T. Faucett, J. Thaler, and D. Whiteson, Phys. Rev. D 103, 036020 (2021).

[54] R. E. Owen (ATLAS Collaboration), https://cds.cern.ch/ record/2302730 (2018).

[55] V. Khachatryan, A. Sirunyan, A. Tumasyan, W. Adam, E. Asilar, T. Bergauer, J. Brandstetter, E. Brondolin, M. Dragicevic, J. Erö et al., J. Instrum. 12, P01020 (2017).

[56] B. T. Huffman, C. Jackson, and J. Tseng, J. Phys. G 43, 085001 (2016).

[57] P. A. Zyla et al. (Particle Data Group Collaboration), Prog. Theor. Exp. Phys. 2020, 083C01 (2020).

[58] E. Witten, Nucl. Phys. B160, 57 (1979).

[59] M. Aaboud et al. (ATLAS Collaboration), Phys. Rev. D 96, 052004 (2017).

[60] M. Aaboud et al. (ATLAS Collaboration), Phys. Lett. B 788, 316 (2019).

[61] A. M. Sirunyan et al. (CMS Collaboration), Phys. Rev. Lett. 119, 111802 (2017).

[62] A. M. Sirunyan et al. (CMS Collaboration), Phys. Lett. B 769, 520 (2017); 772, 882(E) (2017).

[63] M. Aaboud et al. (ATLAS Collaboration), Phys. Rev. Lett. 121, 081801 (2018).

[64] A. Alloul, N. D. Christensen, C. Degrande, C. Duhr, and B. Fuks, Comput. Phys. Commun. 185, 2250 (2014).

[65] C. Degrande, C. Duhr, B. Fuks, D. Grellscheid, O. Mattelaer, and T. Reiter, Comput. Phys. Commun. 183, 1201 (2012).

[66] J. Alwall, R. Frederix, S. Frixione, V. Hirschi, F. Maltoni, O. Mattelaer, H. S. Shao, T. Stelzer, P. Torrielli, and M. Zaro, J. High Energy Phys. 07 (2014) 079.

[67] L. Carloni and T. Sjostrand, J. High Energy Phys. 09 (2010) 105.

[68] L. Carloni and T. Sjostrand, J. High Energy Phys. 09 (2010) 105. 
[69] T. Sjöstrand, S. Ask, J. R. Christiansen, R. Corke, N. Desai, P. Ilten, S. Mrenna, S. Prestel, C. O. Rasmussen, and P. Z. Skands, Comput. Phys. Commun. 191, 159 (2015).

[70] M. Cacciari, G. P. Salam, and G. Soyez, J. High Energy Phys. 04 (2008) 063.

[71] M. Cacciari, G. P. Salam, and G. Soyez, Eur. Phys. J. C 72, 1896 (2012).

[72] M. L. Mangano, M. Moretti, F. Piccinini, and M. Treccani, J. High Energy Phys. 01 (2007) 013.

[73] V. Izzo (ATLAS Collaboration), Technical Report No. ATLUPGRADE-PROC-2020-001, CERN, Geneva, 2020, http:// cds.cern.ch/record/2732959.

[74] ATLAS Collaboration, Technical Reports No. CERNLHCC-2013-018, No. ATLAS-TDR-023, 2013, final version presented to December 2013 LHCC, http://cds .cern.ch/record/1602235.

[75] ATLAS Collaboration, Technical Report No. ATL-DAQPUB-2018-002, CERN, Geneva, 2018, https://cds.cern.ch/ record/2625986.

[76] G. Aad, B. Abbott, D. C. Abbott, A. A. Abud, K. Abeling, D. K. Abhayasinghe, S. H. Abidi, O. S. AbouZeid, N. L. Abraham et al., Eur. Phys. J. C 80, 47 (2020).

[77] M. Aaboud et al. (ATLAS Collaboration), Eur. Phys. J. C 79, 205 (2019).

[78] M. Aaboud et al. (ATLAS Collaboration), Eur. Phys. J. C 79, 375 (2019).
[79] ATLAS Collaboration, Technical Report No. ATL-PHYSPUB-2017-004, CERN, Geneva, 2017, http://cds.cern.ch/ record/2259646.

[80] ATLAS Collaboration, Technical Report No. ATL-PHYSPUB-2018-014, CERN, Geneva, 2018, http://cds.cern.ch/ record/2630973.

[81] ATLAS Collaboration, Technical Report No. ATL-SOFTPUB-2018-001, CERN, Geneva, 2018, https://cds.cern.ch/ record/2630433.

[82] V. V. Gligorov and M. Williams, J. Instrum. 8, P02013 (2013).

[83] ATLAS Collaboration, Technical Design Reports No. CERN-LHCC-99-014, No. ATLAS-TDR-14, CERN, Geneva, 1999, https://cds.cern.ch/record/391176.

[84] M. Capeans, G. Darbo, K. Einsweiller, M. Elsing, T. Flick, M. Garcia-Sciveres, C. Gemme, H. Pernegger, O. Rohne, and R. Vuillermet (ATLAS Collaboration), Technical Reports No. CERN-LHCC-2010-013, No. ATLAS-TDR-19, 2010, https://cds.cern.ch/record/1291633.

[85] A. Hoecker, P. Speckmayer, J. Stelzer, J. Therhaag, E. von Toerne, and H. Voss, Proc. Sci., ACAT2007 (2007) 040 [arXiv:physics/0703039].

[86] G. Aad et al. (ATLAS Collaboration), J. Instrum. 8, P07015 (2013).

[87] C. Cesarotti and J. Thaler, J. High Energy Phys. 08 (2020) 084. 